This item was submitted to Loughborough's Research Repository by the author.

Items in Figshare are protected by copyright, with all rights reserved, unless otherwise indicated.

\title{
Internet of Things home healthcare: the feasibility of elderly activity monitoring
}

PLEASE CITE THE PUBLISHED VERSION

https://doi.org/10.1109/CSCI46756.2018.00179

PUBLISHER

IEEE

VERSION

AM (Accepted Manuscript)

\section{PUBLISHER STATEMENT}

Personal use of this material is permitted. Permission from IEEE must be obtained for all other uses, in any current or future media, including reprinting/republishing this material for advertising or promotional purposes, creating new collective works, for resale or redistribution to servers or lists, or reuse of any copyrighted component of this work in other works.

\section{LICENCE}

CC BY-NC-ND 4.0

\section{REPOSITORY RECORD}

Power, Luke, Sarah Dunnett, and Lisa Jackson. 2019. "Internet of Things Home Healthcare: The Feasibility of Elderly Activity Monitoring". figshare. https://hdl.handle.net/2134/36627. 


\section{Internet of Things Home Healthcare: The Feasibility of Elderly Activity Monitoring}

\author{
Luke Power \\ Aeronautical and Automotive \\ Engineering \\ Loughborough University \\ Loughborough, United Kingdom \\ 1.power@lboro.ac.uk
}

\author{
Sarah Dunnett \\ Aeronautical and Automotive \\ Engineering \\ Loughborough University \\ Loughborough, United Kingdom \\ S.J.Dunnett@1boro.ac.uk
}

\author{
Lisa Jackson \\ Aeronautical and Automotive \\ Engineering \\ Loughborough University \\ Loughborough, United Kingdom \\ L.M.Jackson@1boro.ac.uk
}

\begin{abstract}
Homecare systems are a focus of research due to shifting care requirements of the elderly. Activity has become a vital metric when monitoring vulnerable patients. Activity monitoring however is contextual and difficult to capture. In this paper the implementation of a Bluetooth low energy monitoring system which incorporates the interoperability of Internet of Things (IoT) to create a sustainable homecare approach is explored. Monitored patient health is evaluated using activities of daily living standards. Data captured is evaluated to determine movement, motion and location which contribute to the activity based, sensor driven care models. Activity is captured by occupants interacting with 'unique' objects of interest (OOI). Interactions captured are evaluated using activities of daily living by aligning room positioning, transference within the home and OOI use.
\end{abstract}

Keywords - Internet of Things, Homecare, Activity Monitoring, Lifestyle Monitoring, Indoor Positioning System

\section{INTRODUCTION}

As life expectancy increases due to advancements in medical science and improving living conditions, national healthcare systems have been placed under ever increasing strain to provide elderly care outside of the hospital environment which suffers from over-demand. Technologies researched in this space focus on collecting home patient data through a variety of devices with the objective of predicting complications while having the ability to provide alerts and knowledge of patient wellbeing through data analysis (Stowe \& Harding, 2010). The Internet of Things (IoT) concept may have a place within this scope as internet connected devices currently can be found in over $85 \%$ of UK homes (Office of National Statistics, 2018). However, technology as a homecare solution presents with additional challenges compared to standard care. Devices examined for IoT enabled homecare often require infrastructure to handle sensitive medical data or to integrate with existing healthcare services, this requires extensive setups and continued maintenance placing feasibility of use in doubt (Vegesna et al, 2017). This paper investigates the viability of IoT enabled homecare activity monitoring using proximity-based Bluetooth Low-Energy (BLE) beacons. These devices possess traits which may overcome the challenges which technology in homecare faces.

\section{PREVIOUS WORK}

The growing elderly population requires a persistent standard of care, encompassing many areas of daily life. Research has attempted to define lifelong elderly care as maintaining independence while reducing vulnerability. In their article, Kenneth et al found that over $50 \%$ of the elderly, aged 70 and above were hindered in their ability to maintain activities of daily living (ADL). The authors demonstrated ADL as an accurate predictor of functional decline leading to complications with care and increased hospitalization (Kenneth E et al, 2003). Devices to monitor ADL have limitations which manifest when required to provide care functions for chronic non-communicable diseases (NCD) such as dementia, isolation, sedentary lifestyles, malnourishment and loss of independence (Neri et al, 2012). Patient activity metrics are not easily captured or identifiable variables. Extensively researched monitoring devices typically have limitations in this area of activity monitoring (Vegesna et al, 2017).

\section{METHOD}

This study required two phases of testing to determine the efficacy and feasibility of IoT enabled homecare monitoring using BLE. The first phase tested the suitability of BLE beacons as an accurate device for distinguishing lifestyle events of a home occupant which align most to the ADL standards of independence and health. The second phase evaluated device feasibility in terms of interoperability, flexibility and maintenance of the devices within an IoT smart home network. Phase 1 attempts to demonstrate BLE beacons placed in rooms and on objects of interest can gauge distance between receiver and advertiser accurately while also investigating the ability to observe movement patterns between rooms and interactions with objects thus demonstrating efficacy in IoT devices for homecare monitoring. Phase 2 aims to provide feasibility to sensors for use in homecare by examining signal derogation and battery life with the view to establishing the feasibility of operation in such an environment. Using BLE as a remote indoor positioning system (IPS) to gather activity patterns of elderly patients requires the classification of these observations in home environments, ADL details a set of conditions such as transferring, eating and sedentary levels as a framework to assess home patient health 
and independence. Interactions observed using IPS should align with ADL conditions for physical and mental wellbeing (Katz, 1983). Movement would be probable when BLE beacons observe transfer between rooms, attempts to gain nourishment could be interpreted through kitchen proximity and OOI interactions. By placing BLE devices throughout a home environment, rooms of the home will emit proximity radius as shown in figure 1 . An occupant wearing a receiver, moving and performing actions or interacting with objects will be observed entering and exiting the ranges of devices.

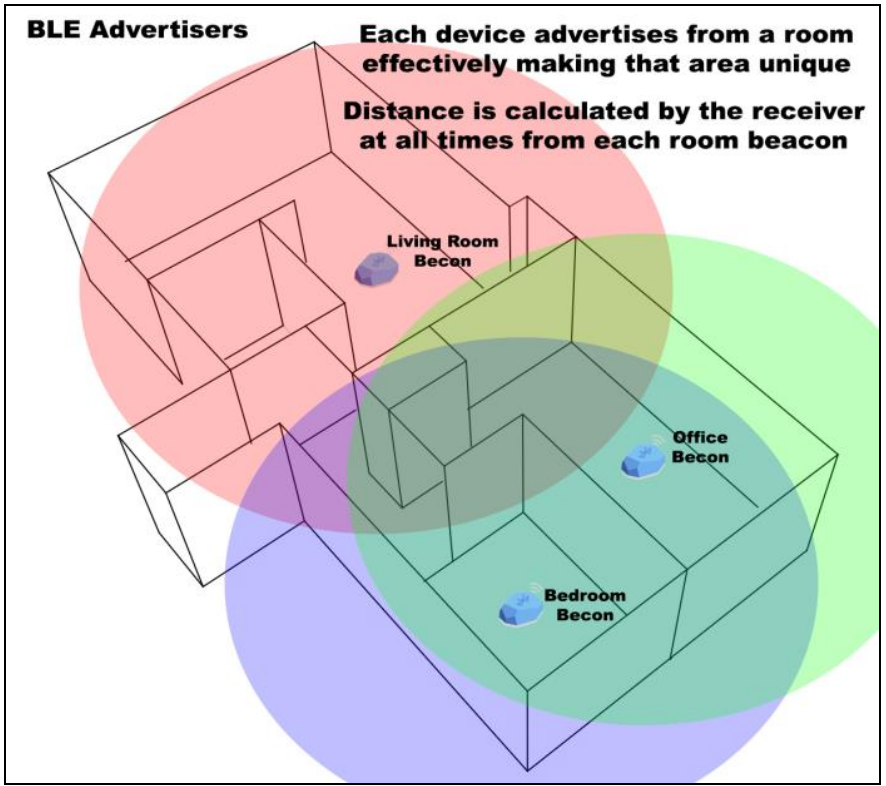

Fig. 1. Proximity Unquie Rooms

Object advertisers are beacons which can be placed on objects of interest such as furniture, kettles, fridges, microwaves or beds, the purpose of which is to make the objects unique in the sensor data to build a pattern of activity. The strongest signal strength to the receiver worn by the patient will indicate the occupant's location at any given time while showing a pattern of movements approximated with BLE devices in rooms and on objects.

\section{Phase 1: FEASIBILITY OF CAPTURE}

In Phase 1 experiments examined distance, pattern recognition and OOI interactions.

\section{A. Accuracy}

Assessing feasibility of any sensor used for monitoring requires establishing acceptable degrees of accuracy, particularly given the potential barriers radio signals experience when operating within closed spaces (Attia et al., 2013). Applying received signal strength indication (RSSI) to distance calculation obtained from Yves et al., when the receiver intercepts a BLE beacon advertisement using RSSI accuracy is established (Joel Yves and Hao, 2015). The transmit power (TX) which produces the highest levels of distance accuracy will determine the most accurate signal output and provide knowledge on the feasible accuracy of BLE devices in the home environment. Additional factors can also influence approximate accuracy outside of TX power, such as advertisement interval and advertisement frequency. Experiments focus on establishing the interval which provides the highest accuracy while not draining battery power excessively.

\section{B. Activity Detection}

Following the establishment of reliable accuracy, further methods captured interactions to recognize activity patterns using ADL. Placing BLE beacons in rooms with functions which could be associated with ADL conditions such as kitchens for nourishment, living rooms and bedrooms for sedentary behavior with movement between these locations indicating transferring. An occupant wearing or carrying the receiver device travels between these rooms while intercepting multiple beacons and leaving signal ranges. Using multiple beacons, the experiment explores intersection of beacon radio signals across the environment to determine likely location by examining distances from each beacon as figure 2 illustrates. With accuracy established in terms of distance it becomes reasonable to conclude the strongest signal received from a BLE device indicates room position. Further experiments sought the ability to recognize patient and location within the setting. This is achieved using distance calculated from RSSI from a single beacon and verified further using additional broadcast points within the smart home.

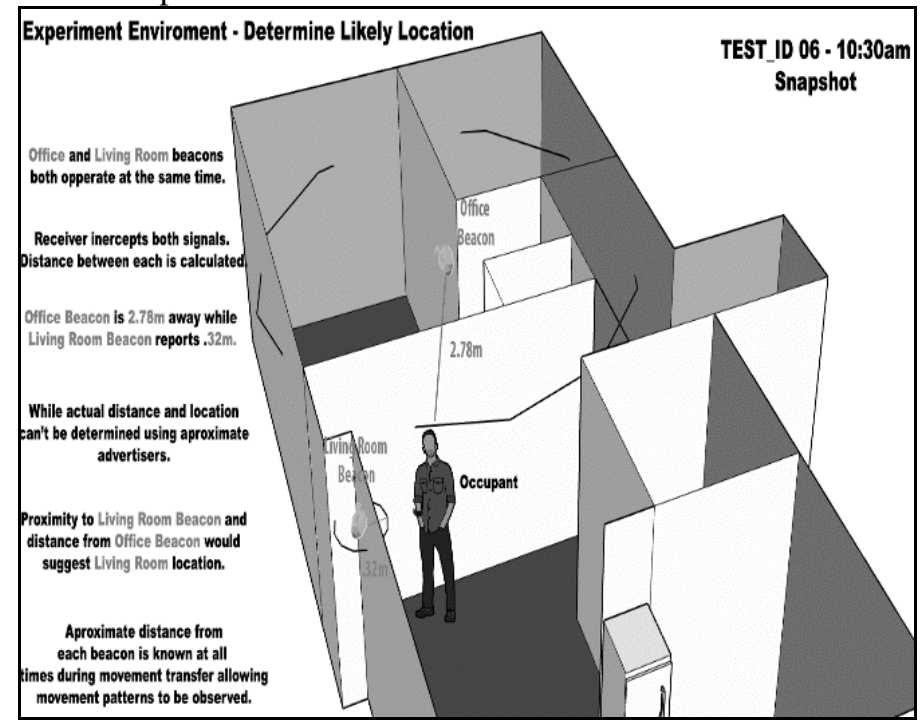

Fig. 2. Proximity Distances

As figure 2 also demonstrates, the occupant is standing nearest to the Living Room Beacon. Validity of location is determined where data points intersect at any given timeframe and likely position of the patient could be determined through process of elimination.

\section{Object Interaction}

Further experiments were performed by placing BLE beacons on objects to create unique objects within a potential IoT smart home and provide additional contextual information 
on how a patient is interacting with their environment. Object use is viable information when used with ADL analysis in determining patient independence and infers the ability for patients to sustain themselves (Pung et al., 2009). Dehydration is one of the leading causes of hospital admission in the UK with efforts of community nurses focused on increasing consumption of water from home care recipients (Gross et al., 1992). Beacons placed on objects of interest such as the fridge, microwave, water bottle and kettle make these objects unique in the environment much like rooms. OOI beacons advertise once they are in motion which indicates manipulation of the object. Motion is interpreted using a gyroscope which is attached to BLE beacons used in this experiment, advertisement only begins once the motion flag is triggered.

\section{Phase 2: Feaseibility In HomeCARE}

\section{A. Settings and procedures}

Phase 2 methods are peformed within a controlled and empty enviroment without any potential barriers to signal advertisement being introduced as part of testing.

\section{B. Battery Life}

Longevity is a factor in device feasibility and maintenance within IoT homecare. All battery life tests measured concurrent usage over a seven day period using the maxium signal strength and advertisement rate available to the hardware. This data was extrapotated into months and years to determine the suitability of Bluetooth low energy in this enviroment given various levels of use including low, optimum operation which is determined from the accuracy experiments and maximum output. Batteries which powered the tested BLE circuit boards contained 1,000 mAh.

\section{EXPERIMENTS \& RESULTS}

Results detailed in this section explore the findings of phase 1 and phase 2 tests. Experiments performed were sampled to determine BLE device accuracy using signal strength, identical experiments are repeated multiple times to eliminate potential variance of signal strength.

\section{A. Equipment}

BLE Receivers including a non-wearable iPhone 7 (IOS, 10.2) and a wearable Sony Smartwatch 3 (Android Wear, 1.5) were used in experiments. BLE devices used to send signals included Estimote Bluetooth Low-Energy Beacons (Eddystone and iBeacon frameworks). The BLE beacons required no specific software while the receiver devices used custom built applications created in JavaScript and Swift respectively. These applications used BLE application programming interfaces (Eddystone, iBeacon) to record variables transmitted by the beacon advertisers. Estimotes brand of BLE beacons include a protective plastic housing, a circuit board featuring a Bluetooth chip, a temperature reader and a force meter powered by a CR2477, 3V Lithium battery with a capacity of 1000 milliamp hours (mhA) rated up to 2 years of standard use. There is no capacity to store data on BLE beacons used. All device interceptions of BLE signals were recorded on the capture devices applications and stored as csv files.

\section{B. Settings and Procedures}

Experiments discussed in this paper take place within a singlestory building with a variety of rooms including a bathroom, bedroom, living room, kitchen and office which simulate a patient's home environment as shown in figure 3. BLE beacons are placed in rooms by their function which identifies with an ADL condition, with living rooms and bedrooms implying sedentary activity while kitchens indicate nourishment attempts and movement between these rooms determining movement transfer.

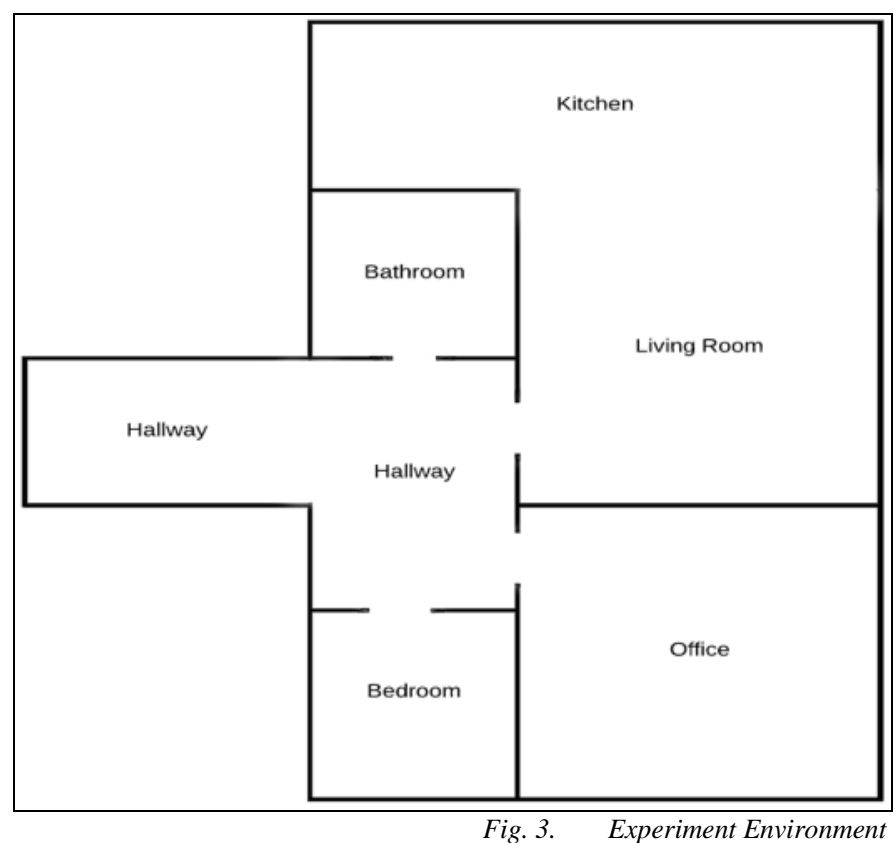

As homecare is part of an overall healthcare system, any device seeking to gain efficacy within the space must be suffiently suitable to the highly variable environment of community healthcare. The primary concerns and limtations of currently available devices are the possiblity of signal derogation and the level of maintinence required to upkeep the technology expressed as battery life.

\section{PHASE 1: RESULTS}

\section{A. Accuracy}

The degree accuracy of the experiments is established using location recognition experiments. Power output is a significant determinant of RSSI, and thus accuracy for radio signals, according to Zhu et al and is reflected in the results of this experiment (Zhu et al., 2005). Frequency of advertisement can also result in a more accurate signal. Table 1 shows the 
accuracy of reported distance vs actual distance when both TX Power and advertisement interval are altered to examine effects of increased accuracy at the cost of longevity. When testing the effect of the advertisement interval, TX Power is taken at its highest power output value of $-4 \mathrm{dbm}$. When testing the effect on accuracy of TX power the advertisement interval was kept constant at $100 \mathrm{~ms}$ as the most frequent advertisement interval possible which insured large amounts of data to analyses with the shortest interval between data points. Ten experiments were performed with each test lasting 10 minutes with the receiver attempting to capture every 1 second resulting in over 1000 individual data points.

TABLE I. ACCURACY WITH VARYING TX POWER

\begin{tabular}{|c|c|c|c|c|}
\hline TX Power & $\begin{array}{c}\text { Advertisement } \\
\text { Interval }\end{array}$ & $\begin{array}{c}\text { Actual } \\
\text { Distance }\end{array}$ & $\begin{array}{c}\text { Average } \\
\text { Captures }\end{array}$ & $\begin{array}{c}\% \text { of } \\
\text { Accuracy }\end{array}$ \\
\hline $4 \mathrm{dbm}$ & $100 \mathrm{~ms}$ & $1 \mathrm{~m}$ & 1000 & $77 \%$ \\
\hline $15 \mathrm{dbm}$ & $100 \mathrm{~ms}$ & $1 \mathrm{~m}$ & 1000 & $70 \%$ \\
\hline $4 \mathrm{dbm}$ & $100 \mathrm{~ms}$ & $1 \mathrm{~m}$ & 1000 & $69 \%$ \\
\hline $4 \mathrm{dbm}$ & $400 \mathrm{~ms}$ & $1 \mathrm{~m}$ & 1000 & $74 \%$ \\
\hline
\end{tabular}

It is not surprising that using higher power outputs and more frequent advertisements results in a more consistent and accurate RSSI which in turns provides a more reliable distance calculation as seen in the 1 st row of table 1 . Frequency of advertisement had little effect on accuracy, as can be seen in the last row of table 1 where a $400 \mathrm{~ms}$ advertisement interval, effectively four times slower than $100 \mathrm{~ms}$, is seen to be $3 \%$ less accurate when compared with the highest rate of $100 \mathrm{~ms}$. Illustrating a snapshot of this result, figure 4 shows a capture of a single beacon, with the erratic blue line showing distance calculated from RSSI in meters while the solid orange line shows the actual distance between the receiver and the beacon.

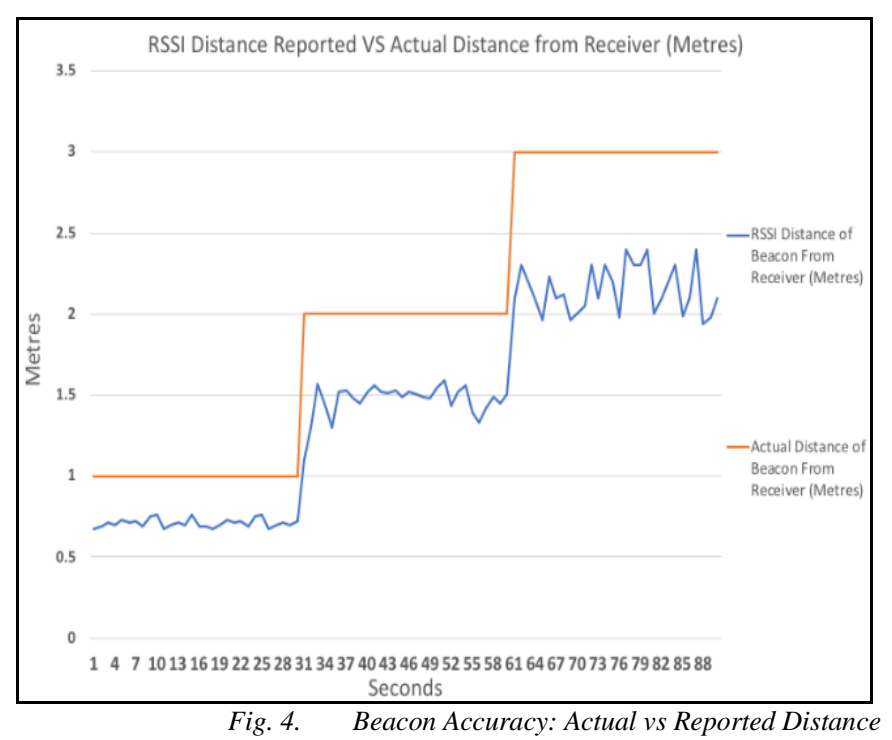

This distance is increased over time during the experiment to monitor the continued accuracy of the beacons (blue line). Ideally the orange and blue lines should be as close as possible which would represent the receiver calculating the correct distance from the broadcasting beacon. The average accuracy of this capture was $77.1 \%$ using $-4 \mathrm{dbm}$ TX Power and $100 \mathrm{~ms}$ advertisement interval. Despite the erratic nature of RSSI it appears advertisers can establish location of a receiver particularly if using multiple sources of confirmation i.e. additional broadcasters. All further tests used $-4 \mathrm{dbm}$ TX Power and $100 \mathrm{~ms}$ advertisement interval.

\section{B. By Room}

Location recognition focused on a single experiment repeated three times to obtain a significant amount of data points to base the analysis. The subject wearing the receiver would move between several rooms in the environment seen in figure 5.

TABLE II. BY ROOM - TIMETABLE OF MOVMENT

\begin{tabular}{|c|c|}
\hline Timeframe & Receiver Location \\
\hline $9: 45-10: 00$ & Office \\
\hline $10: 00-10: 30$ & Bedroom \\
\hline $10: 30-10: 45$ & Living Room \\
\hline $10: 45-11: 00$ & Office \\
\hline
\end{tabular}

Table 2 shows the times the occupant was in each location. Figure 5 shows three beacons operating as blue (Office), green (Bedroom) and yellow (Living Room) for an hour and fifteen minutes. The receiver would move between locations at set times as described in table 2. Travel between rooms can be observed in the intersection points between the lines with overall accuracy and approximate room location distinguishable. Data consistently showed a clearly identifiable nearest beacon using distance in meters in each timeframe.

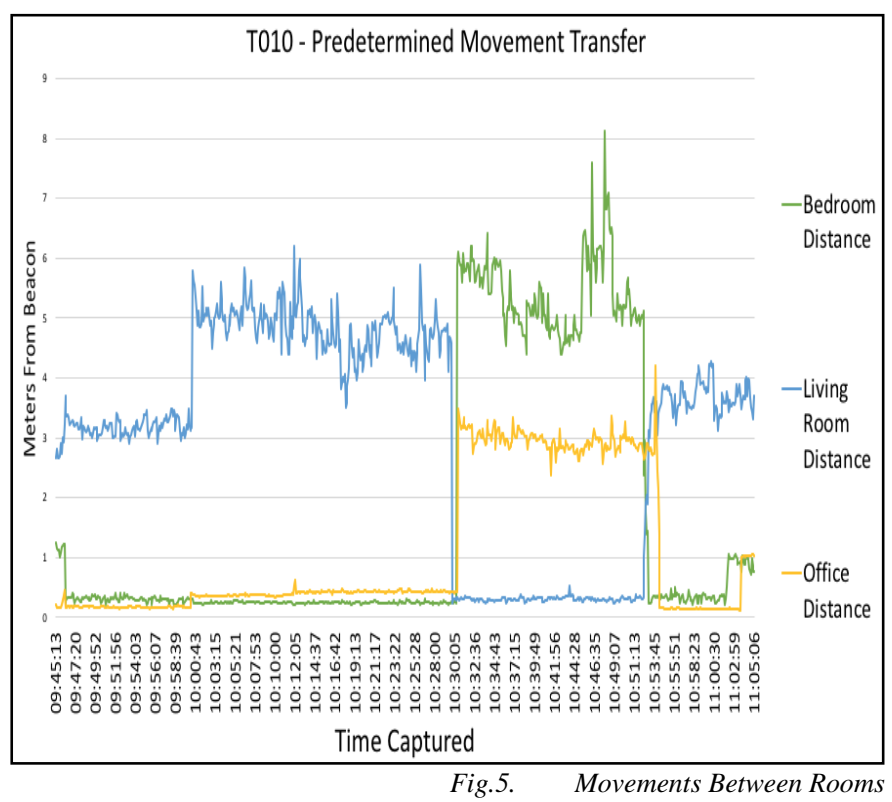

Stationary behavior was also evidently clear, despite fluctuation of RSSI it was possible to establish approximate 
location based on nearest beacon and the process of limitation when comparing to additional beacons which are not 'nearest'.

\section{By Object}

Contextual activity recognition experiments included BLE beacon advertisers placed on objects of interest (OOI). In this experiment, BLE beacons were placed on a fridge and in the kitchen which the fridge sits as table 3 indicates. The BLE fridge beacon is set to advertise only when in motion i.e. the fridge door moving. Results showed efficacy for determining an OOI due to proximity with the advertising beacon when the object is in use.

TABLE III. BY OBJECT - TIMETABLE OF FRIDGE USE

\begin{tabular}{|c|c|c|}
\hline Timeframe & Beacon Location & Action \\
\hline 19:12:45 - 19:18:00 & Kitchen Beacon & Stationary \\
\hline 19:15:00 - 19:15:05 & Fridge Beacon & Fridge Open \\
\hline 19:15:05 - 19:16:44 & Fridge Beacon & Fridge Open \\
\hline 19:16:45 - 19:16:50 & Fridge Beacon & Fridge Closed \\
\hline
\end{tabular}

Operating again for 30 seconds it becomes feasible to confirm the subject wearing or carrying a BLE receiver is interacting with the fridge for more than two minutes within the kitchen. The beacon activates and advertises its RSSI for 30 seconds, it activates again when the door is closed as evidenced in figure 6.

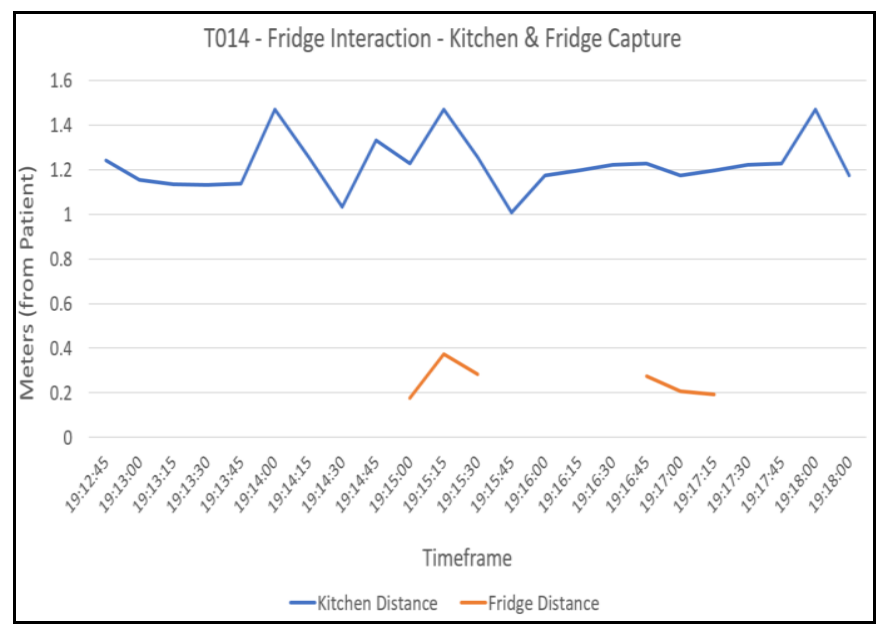

Fig. 6. Fridge Interaction Experiment

\section{PHASE 2 RESULTS}

\section{A. Battery Life Projection}

A new battery was inserted into three beacons with a maximum capacity of $1,000 \mathrm{mhA}$. After seven days use, each device using different power outputs had its remaining capacity measured and extrapolated until the capacity reached empty as shown in figure 7. Advertisement interval had a slight impact on battery life with a modest increase of longevity when extending the interval between signal outputs.
All devices were placed in the same location with two meters of distance between each other to avoid potential interference.

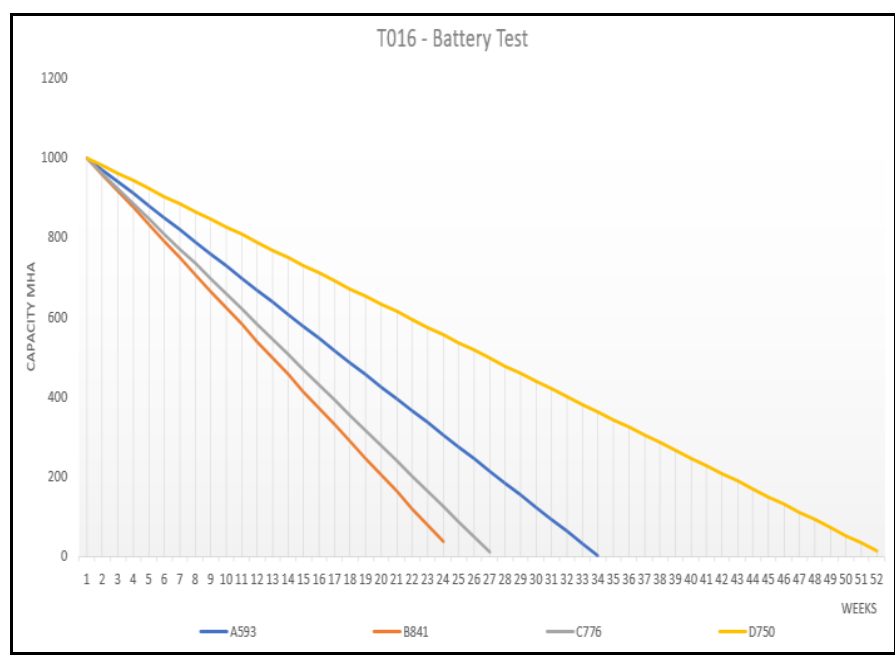

Fig. 7. Battery Life Projections

\section{DISCUSSION}

\section{A. Efficacy in IoT Homecare}

The main strength of the devices determined is the ability to provide robust home care monitoring using a ubiquitous signal with Bluetooth. Consistent accuracy was established with multiple tests allowing for a reliable gauge of RSSI to meter distance in a home environment. Movement events could be clearly examined within data including actions performed on OOI. Battery life tests also showed ability to operate at the highest levels of accuracy for several months, strengthening the case for feasibility.

\section{B. Conclusion}

BLE devices showed efficacy in enabling IoT homecare monitoring by capturing activity data useful in the homecare of elderly patients. Sensors were examined have demonstrated longevity and are interoperable in the increasing world of interconnected devices currently present in the home. The ability to provide elderly patient activity and lifestyle monitoring, factors in the prevention of NCDs form a gap in the research area (Lavizzo-Mourey,1988). This paper has demonstrated the potential feasibility of BLE enabled IoT home care through establishing consistent accuracy, contextual pattern recognition and reaffirming these devices as low maintenance through diminished power output without sacrificing longevity. 


\section{REFERENCES}

[1] Abu-Sharkh, S. and Doerffel, D. (2004). Rapid test and non-linear model characterisation of solid-state lithium-ion batteries. Journal of Power Sources, 130(1-2), pp.266-274.

[2] Attia, M., Moussa, A. and El-Sheimy, N. (2013). Map Aided Pedestrian Dead Reckoning Using Buildings Information for Indoor Navigation Applications. Positioning, 04(03), pp.227-239.

[3] Baker, S. and Quatier, B. (2011). Remote Patient Monitoring. US 20140331298

[4] Barlow, J., Singh, D., Bayer, S. and Curry, R. (2007) 'A systematic review of the benefits of home telecare for frail elderly people and those with long-term conditions', Journal of Telemedicine and Telecare, 13(4), pp. 172-179.

[5] Campling, P. (2014). Intelligent Kindness: professional healthcare and the future of the UK NHS. European Journal for Person Centered Healthcare, 2(2), p.235.

[6] CHENG, S. and LI, J. (2010). ( $(\varepsilon, \delta$ )-Approximate Aggregation Algorithm in Wireless Sensor Networks. Journal of Software, 21(8), pp.1936-1953.

[7] Feil, C. (2016). Indoor Positioning: Opportunities and implementation strategies of Bluetooth Low Energy. GI_Forum, 1, pp.94-105.

[8] Gross, C., Lindquist, R., Woolley, A., Granieri, R., Allard, K. and Webster, B. (1992). Clinical indicators of dehydration severity in elderly patients. The Journal of Emergency Medicine, 10(3), pp.267-274.

[9] Joel Yves, A. and Hao, P. (2015). RSSI-based Indoor Localization Using RSSI-with-Angle-based Localization Estimation Algorithm. International Journal of Sensor Networks and Data Communications, 04(02).

[10] Katz, S. (1983). Assessing Self-maintenance: Activities of Daily Living, Mobility, and Instrumental Activities of Daily Living. Journal of the American Geriatrics Society, 31(12), pp.721-727.

[11] Kyoung Nam Ha Kyung Chang Lee, Suk Lee (2006). Development of PIR Sensor Based Indoor Location Detection System for Smart Home. Journal of Control, Automation and Systems Engineering, 12(9), pp.905-911.

[12] Lavizzo-Mourey, R., Johnson, J. and Stolley, P. (1988). Risk Factors for Dehydration Among Elderly Nursing Home Residents. Journal of the American Geriatrics Society, 36(3), pp.213-218.

[13] Lavizzo-Mourey, R., Johnson, J. and Stolley, P. (1988). Risk Factors for Dehydration Among Elderly Nursing Home Residents. Journal of the American Geriatrics Society, 36(3), pp.213-218.

[14] Lehoux, P. (2004). Patients' perspectives on high-tech home care: a qualitative inquiry into the user-friendliness of four technologies. BMC Health Services Research, 4(1).
[15] Liu, Z., Liu, L. and Barrowes, B. (2010). The Application of the HilbertHuang Transform in Through-wall Life Detection with UWB Impulse Radar. PIERS Online, 6(7), pp.695-699.

[16] Pollock, K., Nichols, J., Simons, T., Farnsworth, G., Bailey, L. and Sauer, J. (2002). Large scale wildlife monitoring studies: statistical methods for design and analysis. Environmetrics, 13(2), pp.105-119.

[17] Pung, H., Gu, T., Xue, W., Palmes, P., Zhu, J., Ng, W., Tang, C. and Chung, N. (2009). Context-aware middleware for pervasive elderly homecare. IEEE Journal on Selected Areas in Communications, 27(4), pp.510-524.

[18] Office for National Statistics. (2018). Mobile phone ownership in the UK - Office for National Statistics. [online] Available at: https://www.ons.gov.uk/aboutus/transparencyandgovernance/freedomofi nformationfoi/mobilephoneownershipintheuk [Accessed 18 May 2017].

[19] Office for National Statistics (2015). Population Estimates for UK, England and Wales, Scotland and Northern Ireland, Mid-2014 - ONS. [online] Available at: http://www.ons.gov.uk/ons/rel/popestimate/populationestimates-for-uk--england-and-wales--scotland-andnorthern-ireland/mid-2014/index.html [Accessed 25 January. 2017].

[20] Office for National Statistics (2016) Deaths registered in England and wales (series DR): $2015 . \quad$ Available at:https://www.ons.gov.uk/peoplepopulationandcommunity/birthsdeaths andmarriages/deaths/bulletins/deathsregisteredinenglandandwalesseriesd r/2015 (Accessed: 08 February 2017).

[21] Stowe, S., \& Harding, S. (2010). Telecare, telehealth and telemedicine. European Geriatric Medicine, 1(3), 193-197. http://doi.org/10.1016/j.eurger.2010.04.002

[22] Nakamura, N., Koga, T., \& Iseki, H. (2013). A meta-analysis of remote patient monitoring for chronic heart failure patients. Journal of Telemedicine and Telecare, 20(1), 11-17. doi: $10.1177 / 1357633 \times 13517352$

[23] Vegesna, A., Tran, M., Angelaccio, M. and Arcona, S. (2017) 'Remote patient monitoring via non-invasive digital technologies: A systematic review', Telemedicine and e-Health, 23(1), pp. 3-17. doi: 10.1089/tmj.2016.0051. WHO (2014) Global health workforce shortage to reach 12.9 million in coming decades. Available at: http://www.who.int/mediacentre/news/releases/2013/health-workforceshortage/en/ (Accessed: 05 February 2017).

[24] WHO (2012) Deaths from NCDs. Available at: http://www.who.int/gho/ncd/mortality_morbidity/ncd_total/en/ (Accessed: 05 February 2017)

[25] Zhu, J. and Durgin, G. (2005). Indoor/outdoor location of cellular handsets based on received signal strength. Electronics Letters, 41(1), p.24. 